\title{
EDUCACIÓN RELIGIOSA ESCOLAR EN CONSTITUCIONES Y LEYES DEL PERÚ REPUBLICANO
}

Religious school education in constitutions and laws of Republican Peru

Carlos Eduardo Palomino Thompson

Universidad Femenina del Sagrado Corazón epalominot40@gmail.com

\section{RESUMEN}

El autor realiza una revisión de las constituciones del Perú, así como de las leyes educativas más importantes de la época republicana, en relación a la enseñanza religiosa escolar. Asimismo, en lo referente al espiritu religioso fundamental de las diversas cartas magnas del Perú.

La larga tradición católica ha dejado una impronta religiosa importante, la misma que se ha traducido en un consenso constitucional y legal en el sentido de que la enseñanza religiosa, en la vida escolar, debe tener presencia curricular y, concretamente, de lo que se llama comúnmente el curso de Religión, en este caso católica, dada la gran mayoría de la población que pertenece a ella, pero respetando la libertad de los padres e instituciones educativas. En las últimas décadas y más, la libertad de cultos constitucional se ha concretado, lógicamente, en la no obligatoriedad de la enseñanza religiosa católica a los estudiantes cuyos padres no lo desean.

Este estudio hace ver que esta línea educativa tiene una estrecha relación con el sentido entero de las constituciones, las cuales expresan también una orientación al menos de mucho apoyo y respeto a lo religioso y, en este caso, fundamentalmente a lo católico.

El autor hace notar, asimismo, que esta relación entre Estado e Iglesia, o si se quiere entre religión y civismo, no tiene carácter fundamentalista como ocurre en la actualidad en diversos estados del mundo. En todo caso podrá haber sido a lo más "regalista" por la elección de los Obispos por el Congreso (Patronato), pero sin que se dieran mayores excesos. La legislación educativa, aunque con ciertas variantes de acuerdo a corrientes nacionales más o menos clericales o anticlericales, ha seguido la misma línea debido a las leyes educativas de estos 200 años, así como a la normatividad.

Palabras clave: Constitución, ley, normativa, educación integral, fundamentalismo, formación religiosa, regalismo, patronato, halo religioso.

\section{ABSTRACT}

The author performs a review of the Constitutions of Peru, as well as the most important educational laws of the Republican era in relation to religious school teaching. Also with regard to the fundamental religious spirit of the various magna letters of Peru.

The long Catholic tradition has left an important religious imprint, which resulted in a constitutional and legal consensus in the sense that religious teaching in school life must have a curricular presence and specifically, what is commonly called the religion course, in this case a catholic course, because the vast majority of the population belongs to it, but respecting parents and educational institutions freedom.

In the last decades and more, the constitutional freedom of cults has been logically concretized in the non-obligatory of religious catholic teaching to students whose parents do not wish it.

This study shows that this educational line has a close relationship with the whole sense of the constitutions, which also express, at least, an orientation of much support and respect for religion, in this case, for the Catholic religion essentially.

The author also notes that this relationship between State and Church, or if you want between religion and civility, is not a fundamentalist one as it is currently in various states of the world. In any case, it may have been a "regalist" for the election of the Bishops by the Congress (Patronage), but without major excesses. The educational legislation, although with certain variants according to national currents more or less clerical or anticlerical, has followed the same line due to the educational laws of these 200 years, as well as to the normativity.

Keywords: Constitution, law, normativity, integral education, fundamentalism, religious formation, regalism, patronage, religious halo. 


\section{INTRODUCCIÓN}

Con cierta frecuencia se escucha decir que el mundo se ha olvidado de Dios, o peor todavía, que se ha puesto en contra de Él y que, por ello mismo, hay una crisis de sentido de la vida y de valores nunca vista. Asimismo: que la ética y sus principios y práctica han quedado relegados; en fin, que en el mundo actual impera un notable relativismo que hace que cada quien se vuelva subjetivista y considere que diversas conductas, incluso contradictorias, pueden ser escogidas dependiendo exclusivamente de la persona. Es decir, no hay una objetividad ética; cada quien piensa y actúa como le parece de acuerdo a su propio sentir al cual convierte en ley.

Pero, de otro lado, también se puede decir que hoy en día existe una creencia bastante generalizada en un Dios creador de todo, pero al mismo tiempo muy poco influyente en las costumbres éticas, morales y cívicas, así como en una espiritualidad que lleve a relacionarse profundamente con la divinidad.

Respecto a la creencia en Dios, una encuesta realizada por WIN internacional con Datum internacional (2017) en 68 países, concluía que en el mundo un $71 \%$ cree en Dios. En el Perú la encuesta señalaba nada menos que un $92 \%$.

La lectura de las constituciones y leyes de esta época republicana del Perú hace ver, en el primer siglo y algo más, una mayor fortaleza y relación entre el contenido constitucional, la fe religiosa, en este caso católica, y la educación escolar religiosa.

En las últimas décadas estos rasgos fundamentales no han desaparecido, pero se han debilitado en cuanto a las constituciones del siglo XIX, como se irá viendo en el transcurso de esta investigación, lo cual parece de alguna manera lógico ante nuevos conceptos de laicidad, separación de poderes y otros.

No obstante, la documentación nacional sigue siendo muy clara, también en la actualidad, en cuanto a la obligación de brindar en la etapa escolar la enseñanza religiosa, particularmente católica, excepto lógicamente en los centros de otras confesiones.
Todo esto obedece a ideas de los tiempos que van debilitando mucho el sentido religioso. Se percibe asimismo una posición opuesta y contestataria de un cierto grupo de personas, y también instituciones, que desearían que esta formación religiosa desapareciera de la escolaridad.

La revisión de lo constitucional y lo legislado hará ver la clara obligación de la enseñanza religiosa escolar en el Perú, la cual existe, ha existido y existe en Perú, así como su sentido integrador con lo cívico y ciudadano, dentro de un concepto de formación integral propuesto por la Constitución vigente de 1993.

\section{LASCONSTITUCIONESYLAENSEÑANZA RELIGIOSA ESCOLAR}

Las constituciones del primer siglo de la República tienen una mayor cercanía a lo religioso, digamos un cierto "halo religioso" que las ilumina como fondo de lo que expresan, así como un concepto de "educación integral" que une mucho lo ético, cívico y religioso. Asimismo una claridad absoluta de la obligatoriedad de la enseñanza religiosa escolar de orientación católica. El último siglo educativo constitucional y legal también afirma buena parte de ello en cuanto a la enseñanza religiosa escolar, pero de manera más débil y, lógicamente dentro de un concepto de libertad religiosa.

\section{- LA CONSTITUCIÓN DE CÁDIZ (1812)}

Esta constitución de la monarquía española, de los tiempos inmediatos previos a la independencia, tuvo un claro corte liberal, pero apenas un par de años de duración. La participación de peruanos y americanos, tanto en las votaciones de diputados como con la presencia de representantes de este continente en el Congreso Constituyente, causó interés y despertó ánimo y esperanzas.

Esta Constitución influyó mucho en todo el continente y de manera especial en nuestra primera carta constitucional de 1823. De allí la importancia de hacer un recuento de sus aportes en las líneas propuestas de estudio.

Lo que podríamos llamar el "halo religioso", y concretamente católico, se muestra en una serie de artículos. 
Por ejemplo, la Constitución de 1812 se inicia así: "En el nombre de Dios Todopoderoso, Padre, Hijo y Espíritu Santo, autor y Supremo Legislador de la sociedad".

El artículo 12 dice: "La religión de la Nación española es y será perpetuamente la católica, apostólica y romana, única verdadera. La Nación la protege por leyes sabias y justas, y prohibe el ejercicio de cualquier otra".

Los artículos 47, 48, 58, 71, 86, 87, 91, 117, 155,173 y 212 , referidos a juramentaciones de representantes en elecciones, o del Rey y príncipe de Asturias, hacen alusión a que se hacen a Dios y para que Él demande su incumplimiento. Asimismo varios de ellos aluden a la Misa del Espíritu Santo como acto integrador y relevante.

En cuanto a la obligatoriedad de la enseñanza religiosa, el artículo 366 se expresa así: "En todos los pueblos de la monarquía se establecerán escuelas de primeras letras, en las que se enseñará a los niños a leer, escribir y contar, y el catecismo de la religión católica, que comprenderá también una breve exposición de las obligaciones civiles".

Por lo que se refiere al sentido de una educación de alguna manera integral, al menos procurando unir lo civil y religioso, las dos últimas líneas del párrafo anterior son muy claras.

\section{- LA PRIMERA CONSTITUCIÓN DE LA REPÚBLICA (1823).}

La primera Constitución de Perú está considerada en el grupo de las de tendencia liberal. Su gran extensión muestra que se quisieron establecer muchas cosas y con precisión.

Lo que se está considerando como el "halo religioso" de las constituciones del Perú está muy claramente expresado en los artículos 8 y 9 y algo en el 52. Así: "La religión de la República es la Católica, Apostólica y romana, con exclusión de ejercicio de cualquier otra" (Art. 8).

"Es un deber de la nación protegerla constantemente, por todos los medios conforme al espiritu del Evangelio $y$ de cualquier habitante del Estado representarla inviolablemente" (art. 9).
El art. 52 se refiere al tradicional juramento religioso de los diputados.

En cuanto a la enseñanza de la Religión, aparte del artículo 90, inciso10, que hace referencia a que en las reducciones de los Andes se había de "promover la civilización y conversión de los infieles conforme al espíritu del Evangelio", se dice en el artículo 184:

"Todas las poblaciones de la República tienen derecho a los establecimientos de instrucción que sean adaptables a sus circunstancias. No puede dejar de haber Universidades en las capitales de departamento, ni escuelas de instrucción primaria en los lugares más pequeños; la que comprenderá también el catecismo de la Religión Católica y una breve exposición de las obligaciones morales y civiles".

En este caso, otra vez unido lo referente a lo religioso y lo civil con un sentido de integralidad o una línea de educación integral, al menos en dos vectores importantísimos de ella.

\section{- CONSTITUCIÓN DE 1828}

La Constitución de 1828, considerada liberal, va a ser más bien relativamente corta comparada con muchas otras.

Si bien el "halo religioso" es claro: al invocarse a Dios Todopoderoso; al establecer que la Religión es la católica (art.3); en el juramento central presidencial en nombre de Dios; así como otras relaciones entre el Congreso y los nombramientos eclesiásticos, no hay alusión alguna a la enseñanza de la Religión en las escuelas, lo cual se puede explicar por el escaso espacio dado a la educación en la Constitución y la mayor brevedad de ésta comparada con la de 1823.

\section{- CONSTITUCIÓN DE 1834}

La Constitución de 1834 fue también de tendencia liberal, fue corta e hizo cambios mínimos a la de 1828 .

Manifiesta que la Religión católica es la de la Nación. Su "halo religioso" es claro aunque menor que las anteriores. En cuanto al tema de la educación, apenas le da un artículo de menos de 
tres líneas, con lo que la alusión a la enseñanza religiosa no aparece.

La relación con la Iglesia Católica sí es visible en el manejo regalista de nombramientos episcopales mediante el régimen del Patronato, aprobación de Bulas, etc.

\section{- CONSTITUCIÓN DE 1839}

Fue la 5ta. Constitución del Perú, siendo Presidente Provisorio el general Agustín Gamarra. Con frecuencia es llamada la Constitución de Huancayo y fue de orientación conservadora y centralizadora. Tiene un articulado similar a las anteriores, siendo eso sí, mucho más breve que la primera de 1823.

$\mathrm{Su}$ "halo religioso" se muestra desde su inicio "En el nombre de Dios...", así como el casi inmediato título II, artículo 3, referido a la profesión en el Perú de la religión católica sin permitir el ejercicio público de otra. Dentro de esta línea se dice que el Presidente de la República no puede permitir el ejercicio de otro culto.

No hay alusión alguna a la enseñanza de la Religión en las escuelas. El tema educativo es apenas aludido muy brevemente en los artículos 55 y 174 en el sentido de garantizar la educación Primaria gratuita y que el Congreso organiza los planes y programas de instrucción pública.

\section{- CONSTITUCIÓN DE 1856}

La Constitución de 1856, que duró solamente cuatro años, se inició con un tono algo más bajo en lo que sería su "halo religioso" comparado con las anteriores: "Bajo la protección de Dios..." (previo al artículo 1).

No obstante, este sentido religioso no quita la injerencia del Estado, como en las constituciones anteriores, en los nombramientos episcopales, pase a Bulas pontificias, y otros, bajo el tradicional régimen del "Patronato", heredado de la época virreinal y que esta Constitución lo especifica en el artículo Nro.89, inciso 15, cuando menciona entre las atribuciones del Presidente de la República: "Ejercer el Patronado con arreglo a las leyes y práctica vigente".
No obstante, de inmediato repite el conocido artículo (Nro. 4) de otras constituciones anteriores: "La nación profesa la Religión Católica, Apostólica, Romana. El Estado la protege por todos los medios conforme al espíritu del Evangelio y no permite el ejercicio público de otra alguna".

En la parte educativa se establecen dos artículos solamente: el 23 donde se garantiza la instrucción obligatoria y gratuita y el 24 que manifiesta el ejercicio libe de la enseñanza y la dirección de los establecimientos de educación.

Por tanto no hay referencia alguna a la educación religiosa, como tampoco a aspecto alguno de sus finalidades cívicas o de logros educativos como lectura, escritura etc., es decir sobre una educación integral.

Se dice que el espíritu liberal de la Constitución y sus discusiones internas fueron menos favorables a lo católico.

\section{- CONSTITUCIÓN DE 1860}

La constitución de 1860 está considerada como moderada y fue fruto de mucho trabajo para lograr consensos entre liberales y conservadores. Fue promulgada por el presidente Ramón Castilla.

Salvo algunos intermedios por diversas causas fue la Constitución más duradera de la época republicana: de 1860 al 1920.

$\mathrm{Su}$ "halo religioso" católico se muestra desde el principio más suave que en las primeras constituciones y se inicia con un escueto: "Bajo la protección de Dios". Pero rápidamente, en el artículo 2, se vuelve a la antigua claridad: "La Nación profesa la Religión Católica, Apostólica, Romana: el Estado la protege, y no permite el ejercicio público de otra alguna". De otro lado, las limitaciones a la Iglesia Católica mediante el Patronato seguían muy fuertes y por tanto la injerencia política en lo religioso.

En cuanto a la enseñanza de la Religión en las escuelas, no hay ninguna referencia. Para la educación se repiten únicamente los dos cortos artículos de la Constitución de 1856 ya mencionados. Tampoco se observa algo en lo referente a una educación integral que al menos una lo cívico con lo religioso. 


\section{- CONSTITUCIÓN DE 1867}

Esta Constitución, la octava, tuvo apenas cuatro meses de duración. Esto se debió en gran parte a su carácter muy liberal al punto que se ha dicho que ha sido la más liberal de la República. Trató de seguir, y profundizar, la liberal de 1856 en la que tenía mucha fuerza el legislativo en contra de la vigente de 1860 en la que el ejecutivo tenía mejor posición, ya que se había llegado a un consenso de moderación luego de arduo trabajo. Por ello fue rápidamente cuestionada y tuvo tan breve duración, volviéndose a la de 1860 .

Se inicia con la conocida frase "Bajo la protección de Dios". En el artículo 3ro se vuelve a aprobar que "el Estado la protege y no permite el ejercicio público de otra alguna". Esta última expresión fue aprobada apenas por tres votos de mayoría y expresaba el sentido liberal y con frecuencia anticlerical de muchos asambleístas. Por ello mismo no hay una en la Constitución algo referente a la "enseñanza religiosa" escolar y el "halo religioso" de la misma existe, pero es débil.

\section{- CONSTITUCIÓN DE 1920}

Esta Constitución se elaboró a la sombra del golpe de Estado de Leguía, al sospechar que no se iba a aprobar su legítima elección como Presidente, así como ante su deseo de tener una Carta Magna a su medida y un Congreso que no se le opusiera y que permitiera las reformas que deseaba dentro un gobierno de carácter "progresista".

La Constitución se inicia invocando los "sagrados nombre de Dios y de la Patria". E1 artículo 5to dice: "La Nación profesa la Religión Católica, Apostólica, Romana. El Estado la protege”. Ya no se habla como en las anteriores de: "no permite el ejercicio público de otra alguna",

Dentro de este giro adecuado el artículo 23 dice: "Nadie podrá ser perseguido por razón de sus ideas ni por razón de sus creencias".

De otro lado, los artículos 53 y 54 no hacen referencia a la obligación de la enseñanza religiosa, sino a aspectos más generales como en las constituciones inmediatamente anteriores, donde lo referente a la enseñanza religiosa se trasladó a las leyes de Educación. Los artículos que se refieren a controles de la Iglesia por el Estado, como el Patronato, siguieron como en las constituciones anteriores. Desde luego que los juramentos en nombre de Dios desaparecieron quedando para el protocolo interno. En síntesis no se puede negar que el "halo religioso" constitucional persiste pero más suave y menos exigentemente católico.

\section{- CONSTITUCIÓN DE 1933}

Sin el tradicional inicio que invoca a Dios, esta décima Constitución del Perú (1933) se elabora en momentos difíciles y contestatarios, luego de la caída de Leguía. Otorga muchos poderes al Legislativo ante los temores de nuevas dictaduras encabezadas por el Ejecutivo.

Los tiempos son más laicos y contestatarios y los muchos constituyentes también, lo que propicia un "halo religioso" menor, aunque los fundamentos religiosos y católicos continúan vigentes.

Así: "La libertad de conciencia y de creencia es inviolable. Nadie será perseguido por razón de sus ideas" (art. 59). Se sigue limitando, controlando y quitando libertad a la Iglesia Católica en nombramientos y otros (artículos 123, 154, 232 y 235).

No obstante, queda un artículo que dice: "Respetando los sentimientos de la mayoría nacional, el Estado protege la Religión Católica, Apostólica, Romana. Las demás religiones gozan de libertad para el ejercicio de sus respectivos cultos" (art. 232). Es evidente que el clima había cambiado, pero tampoco era adverso.

En cuanto a la "enseñanza religiosa", ella no figura y queda más bien ligada a las leyes educativas como se verá en su momento.

\section{- CONSTITUCIÓN DE 1979}

La Asamblea Constituyente que elaboró la Constitución Política promulgada por ella en julio de 1979, la inició con un hermoso Preámbulo que dice así: "Nosotros, Representantes a la Asamblea Constituyente, invocando la protección de Dios y en ejercicio de la potestad soberana que el pueblo del Perú nos ha conferido...". Un inicio que le otorga un "halo religioso" del que careció la anterior.

Luego en el segundo artículo, tercer inciso, al establecer los derechos de toda persona, manifiesta: 
"A la libertad de conciencia y de religión, en forma individual o asociada. No hay persecución por razón de ideas o creencias. El ejercicio público de todas las confesiones es libre, siempre que no ofenda a la moral o altere el orden público". Asimismo: "A guardar reserva sobre sus convicciones políticas, filosóficas y religiosas o de cualquier otra indole" (inciso 17).

El capítulo IV, dedicado a la Educación, la Ciencia y la Cultura en su artículo inicial, el 21, dice: "La Educación tiene como fin el desarrollo integral de la personalidad". Es evidente que siendo lo religioso un elemento de ella, una educación religiosa es parte de una formación integral.

El artículo 22 es central en cuanto a la "enseñanza religiosa" en el ámbito escolar. Dice así: "La formación ética y cívica es obligatoria en todo el proceso educativo. La educación religiosa se imparte sin violar la libertad de conciencia. Es determinada libremente por los padres de familia. La enseñanza sistemática de la Constitución y de los derecho humanos es obligatoria en los centros de educación civiles y militares y en todos sus niveles". De todo ello se deduce que lo ética, cívico, religioso, constitucional y de derechos humanos debe figurar en el desarrollo escolar y otros.

En el capítulo I del título III, cuando se habla del Estado, el artículo 86 dice así: "Dentro de un régimen de independencia y autonomía, el Estado reconoce a la Iglesia Católica como un elemento importante en la formación histórica, cultural y moral del Perú. Le presta su colaboración. El Estado puede establecer formas de colaboración con otras confesiones".

Es cierto que la situación ya no es la de los tiempos iniciales de la República, podría decirse que era exagerada para la Iglesia Católica y hasta injusta para otros, pero es visible que en esta Constitución lo religioso en general está muy garantizado y también la educación religiosa.

Un aspecto muy importante, y que tiene que ver con el clima de buen entendimiento entre el Estado y la Iglesia Católica, es el que se refiere a la desaparición del Patronato y de otras medidas que complicaban las relaciones y a las cuales se ha hecho alusión al comentar otras constituciones. Se concretó en esos mismos años el Acuerdo entre la
Santa Sede y el Estado Peruano que se firmó en julio de 1980. Se hará una alusión a esto más adelante. Sin muchas palabras y juramentos se allanaron muchos caminos y se logró abrir puentes para un mejor entendimiento.

\section{- CONSTITUCIÓN DE 1993}

La Constitución de 1993, vigente, mantiene más del $90 \%$ de lo expresado en los párrafos anteriores en referencia a la de 1979 en cuanto a los temas que se están tratando.

A solamente 14 años de distancia de la Constitución anterior y con una reafirmación de todo lo importante mediante el Acuerdo entre la Santa Sede y el Estado Peruano, era lógico que los cambios fueran mínimos.

Se mantenía por tantola separación colaborativa entre las iglesias y el Estado, en particular la Iglesia Católica. Asimismo el hecho de impartir la educación religiosa en lo escolar, pero con libertad de los padres para excluir a sus hijos de ella si así lo deseaban. Por supuesto el Patronato era ya cosa de la historia, manteniéndose la adecuada separación entre Iglesia y Estado.

También se habla de la integralidad de la educación, lo que obviamente comprende lo religioso.

En el fondo, aunque con pocas expresiones externas como en las primeras constituciones, se tiene una relación Estado-iglesias e Iglesia CatólicaEstado muy independiente, respetuosa y favorable. Los artículos principales expresan lo siguiente:

Preámbulo "El Congreso Constituyente del Perú, invocando a Dios Todopoderoso, obedeciendo al mandato del pueblo..."

"La educación religiosa se imparte con respeto a la libertad de las conciencias" (art. 14).

"Dentro de un régimen de independencia y autonomía el Estado reconoce a la Iglesia Católica como elemento importante en la formación histórica, moral y cultural del Perú y les presta su colaboración, El Estado respeta a otras confesiones y puede establecer formas de colaboración con ellas" (art. 50). 


\section{- ACUERDO ENTRE LA SANTA SEDE Y EL ESTADO PERUANO (1980)}

En la parte final del gobierno del general Francisco Morales Bermúdez, la Santa Sede, cuyo Nuncio era el Arzobispo Mario Tagliaferri, y el Estado peruano, mediante su Ministro de Relaciones Exteriores Embajador Arturo García García, elaboraron y firmaron un Acuerdo que estableció el nuevo sistema de relaciones institucionales entre la Iglesia Católica y el Estado, cosa que resolvió algunos importantes asuntos pendientes y permitió el avance de la excelente relación y aportes entre el Estado y la Iglesia. Aspectos relacionados con el tema que ocupa este artículo, fueron:

- La Iglesia Católica en el Perú goza de plena independencia y autonomía, así como de personería jurídica.

- Los nombramientos episcopales, y otros, se avisarán al presidente previamente para su reconocimiento. Desaparecía el antiguo Patronato y ya no entraban cámaras legislativas y el Ejecutivo en la selección de Obispos y Arzobispos.

- El sistema de subvenciones para las personas, obras y servicios de la Iglesia continuaría como hasta ahora (muchas son educativas comprendiendo los nombramientos de profesores de educación religiosa en las instituciones educativas secundarias pertenecientes al Estado), así como centros escolares particulares subvencionados mediante plazas estatales, lo que facilita pensiones reducidas para los padres de familia.

- "La Iglesia tiene plena libertad para establecer centros educacionales de todo nivel de conformidad con la legislación nacional, en el ámbito de la educación particular... Para el nombramiento de los profesores de Religión Católica de los centros educacionales públicos, en los que continuará impartiéndose, como materia ordinaria la enseñanza religiosa, se requiere presentación del Obispo respectivo (art. XIX).

Al tratarse de un Acuerdo Internacional tiene el valor de un Tratado y ello ha reafirmado y facilitado la colaboración entre la Santa Sede y el Estado Peruano, reforzado lo constitucional expresado anteriormente.

\section{LEYES EDUCATIVAS Y ENSEÑANZA RELIGIOSA ESCOLAR}

La revisión de varias constituciones ha hecho ver la importancia otorgada a la enseñanza religiosa en el mundo escolar y, si en varios casos no figura explícitamente, sí está claramente considerada en leyes educativas de la época. Brevemente se hará alusión a artículos de leyes o reglamentos, con rango de ley de estos dos siglos republicanos, que se refieren a ello: De Ramón Castilla (1850 y 1855); de Manuel Pardo (1876); de Manuel Prado (1941); de Juan Velasco (1972); Fernando Belaunde (1982); de Alejandro Toledo (2003).

\section{- REGLAMENTO DE INSTRUCCIÓN PÚBLICA PARA LAS ESCUELAS Y COLEGIOS DE LA REPÚBLICA. (1850).}

Art. 4: "En toda escuela o colegio se comunicará educación moral y religiosa, cuidándose por quienes corresponda de la pureza de la doctrina y efectividad de la enseñanza".

Art. 7: "En las escuelas de primer orden se enseñará... Catecismo de la religión...".

Art. 8: "En las escuelas de segundo orden se enseñará... religión..."

Art. 12: "En los colegios habrá un Rector...un Capellán destinado a dirigir los actos de culto y comunicar la instrucción religiosa..."

\section{- REGLAMENTO DE INSTRUCCIÓN (1855)}

En los artículos 16 y 17, referidos a la educación popular y a las escuelas de primeras letras, se dice que se dará Religión, Catecismo religioso y político.

En el artículo 27 se dice que ningún alumno podrá ser admitido en los colegios sin ser aprobado en Doctrina Cristiana, y otros. En el 28 se manifiesta que en la enseñanza de los colegios se dará Religión, entre otros. En los colegios nacionales habrá un capellán (art. 33).

\section{- REGLAMENTO GENERAL DE INSTRUCCIÓN PÚBLICA (1876)}

La decidida política educativa del presidente Manuel Pardo se tradujo también en un reglamento general, con carácter de ley, como los de Ramón Castilla. 
Su contenido es muy similar a estos últimos y la preocupación por la "enseñanza de la religión" es clara y la establece en sus diversas etapas. La preocupación por el nombramiento de capellanes y su función de instructores religiosos en los colegios de secundaria es también muy explícita. A continuación algunas citas o referencias.

En el artículo 17, referido a los diversos grados de primaria, se especifica materias religiosas que deben ser enseñadas en sus distintas etapas: doctrina cristiana; historia sagrada; vida de Jesús.

El artículo 35 no deja de ser curioso por su preocupación religiosa y prolijidad cuando establece para los preceptores de las escuelas públicas: "Concurrir con los alumnos que aún no han hecho la Primera Comunión, exceptuando los no católicos, a las explicaciones de la doctrina cristiana que los párrocos deben hacer en sus iglesias".

En el artículo 98 se hace mención a que en la inspección de las escuelas se debe revisar, entre otros, que no se enseñen cosas contrarias a la religión, moral y formas de gobierno.

Los artículos 110, 128, 135 y 141 hacen referencia a los capellanes de los colegios de media y su labor de instrucción en ellos.

\section{- LEY ORGÁNICA DE EDUCACIÓN Nro. 9359 (1941)}

Esta extensa ley abarcaba todo lo educativo y profesoral, incluso lo universitario, por lo que comprendía 658 artículos.

La ley hace muy poca referencia a la enseñanza religiosa, comparando con leyes anteriores, pero es muy clara en cuanto a su enseñanza. Así, en los artículos 93, y 95, en el título de las disposiciones generales, dice:

"La enseñanza estará informada por el espíritu de la doctrina cristiana" (93).

"La educación religiosa es obligatoria en todos los establecimientos de enseñanza primaria y secundaria. Los que pertenezcan a religiones disidentes podrán ser exonerados de dicha enseñanza, previa solicitud por escrito de sus padres o apoderados en el acto de la matrícula" (95).
En el artículo 372, y referidos a centros particulares, se dice: "No se podrá autorizar la apertura de establecimientos de enseñanza como medio de propaganda de religiones disidentes o de ideologías incompatibles con nuestra organización política".

\section{- LEY GENERAL DE EDUCACIÓN Nro. 19326 (1972)}

La discutida ley llamada de la "Reforma Educativa" de la época del general Velasco promulgó una ley, no producto del Congreso sino de un organismo interno gubernamental.

Se cuestionó mucho en su tiempo su sentido estatista-vertical, su orientación socialista y su cierto choque u olvido de lo espiritual religioso, así como el de querer utilizar la educación para un proyecto propio de sociedad.

Por todo ello, las alusiones a lo religioso, ético y afines son débiles y no prioritarias. No obstante, lo que se refiere a la educación religiosa es claro y su ejecución se mantuvo así durante el período de los años 70. Algunos artículos:

"La educación peruana tiene como finalidad fundamental la formación integral de la persona humana en sus proyecciones inmanentes $y$ trascendentes" (art. 6).

"El Estado garantiza y facilita la realización de la educación religiosa en los varios niveles tomando en consideración la realidad socio-religiosa del país $y$ respetando la plena libertad de conciencia, las convicciones de los padres de familia y las posibilidades de opción del educando" (art. 18).

En cuanto a los objetivos de la Educación Básica, en el artículo 92 inciso d se dice:

"Estimular en el educando el sentido de la vida familiar y el espíritu de solidaridad y cooperación social, así como la capacidad para la libre realización de los valores vitales, éticos, religiosos, estéticos y otros...".

Por otra parte, en este mismo gobierno, las diversas confesiones religiosas trabajaron con el gobierno un documento, luego normado oficialmente, con orientaciones para aspectos litigiosos en cuanto a lo religioso en los centros 
educativos y sobre todo a aspectos curriculares, documento que se ha mantenido como un referente seguro y de consenso.

En los años 2010 y 2016 se publicaron ley y reglamento de libertad religiosa los cuales especificaron adecuadamente aspectos de dicha área que, en gran parte, ya estaban claros, pero que fueron mejor precisados.

\section{- LEY GENERAL DE EDUCACIÓN Nro. 23384 (1982)}

La ley de 1982 concreta en el marco educativo lo establecido en la Constitución de 1979-1980 la cual modificaba diversas líneas del gobierno militar 1968-1980.

Esta ley, más breve que las anteriores y posteriores del último siglo, parte en todo momento de una formación integral como objetivo claro de la educación peruana.

Por lo mismo no es muy explícita, aunque sí muy clara, en cuanto el asunto de la enseñanza religiosa escolar. Así, en el artículo 16 dice: "La educación religiosa se imparte como elemento formativo de la personalidad del educando, sin violar la libertad de conciencia del educandos y los educadores. La educación religiosa es determinada libremente por los padres de familia o por los educandos que sean mayores de edad. El Estado colabora con la educación católica de conformidad con el Acuerdo vigente con la Santa Sede. Las diversas confesiones coordinan suacción educativa con el Ministerio de Educación".

En el artículo 4 se excluye todo tipo de discriminación, incluida la religiosa, bajo pena de sanción. En el artículo 41 inciso d, entre los objetivos de la educación Primaria se establece: "Promover el conocimiento y práctica de los valores éticos, cívico-patrióticos, estéticos y religiosos". El mismo concepto se establece para la Educación Primaria de adultos.

Es visible que en las dos décadas en que estuvo vigente esta ley, la educación religiosa fue, como de costumbre, clara en los planes de estudio o el currículo escolar.

\section{- LEY GENERAL DE EDUCACIÓN Nro. 28044} (2003)

En el artículo 6 se habla con énfasis y claridad de la obligatoriedad de la formación ética y cívica a tenor del artículo constitucional correspondiente. Nada se dice en cambio de la educación religiosa de la que trata el mismo artículo constitucional. Ello se deja para el artículo 9 en que de manera más difuminada se dice en el inciso a) al establecer los principios de la educación: "Formar personas capaces delograrsurealizaciónética, intelectual, artística, cultural, afectiva, fisica, espiritual, religiosa, promoviendo...".

Los artículos 29 y 31 tienen referencia a la educación integral y aspectos cercanos a los religiosos, pero parece hubo temor a ser más claros, pese al aval de la Constitución. De todas formas, el artículo constitucional 14: "la educación religiosa se imparte con respeto a la libertad de los educandos" está por encima de la ley y es muy claro". El currículo escolar lo ha considerado y mantiene la educación religiosa como obligatoria en la Educación Básica, aunque hubo momentos en que algunos pretendieron quitarla.

\section{DOCUMENTACIÓN INTERNACIONAL}

- LEGISLACIÓN DE LA IGLESIA CATÓLICA SOBRE LA ENSEÑANZA RELIGIOSA Y LAS ESCUELAS CATÓLICAS (1983) Y PACTO INTERNACIONAL DE DERECHOS ECONÓMICOS, SOCIALES Y CULTURALES DE LAS NACIONES UNIDAS (1966)

Parece conveniente hacer referencia a continuación a algunos artículos del Derecho Canónico así como a otros de Pacto Internacional de Derechos Económicos, Sociales y Culturales referentes a temas tratados en este artículo.

\section{Derecho Canónico“.}

"También tienen los derechos los padres a que la sociedad civil les proporcione las ayudas que necesitan para procurar a sus hijos una escuela católica" (Canon 793,2).

"Como la verdadera educación debe procurar la formación integral de la persona humana, en orden a su fin último, y simultáneamente al bien común de la sociedad, los niños y los jóvenes han de ser educados de manera que puedan desarrollar armónicamente 
sus dotes fisicas, morales e intelectuales, adquieran un sentido más perfecto de la responsabilidad y un uso recto de la libertad y se preparen a participar activamente en la vida social" (Canon 795).

"Deben esforzarse los fieles para que, en la sociedad civil, las leyes que regulan la formación de los jóvenes provean también a su educación religiosa y moral en las mismas escuelas, según la conciencia de los padres" (Canon 799).

"La Iglesia tiene derecho a establecery dirigir escuelas de cualquier materia, género y grado. Fomenten los fieles las escuelas católicas, ayudando, en la medida de sus posibilidades, a crearlas y sostenerlas" (Canon 800).

- PACTO INTERNACIONAL DE DERECHOS ECONÓMICOS, SOCIALES Y CULTURALES (Aprobado por las Naciones Unidas en 1966 y firmado también por el Perú)

3. "Los Estados Partes en el presente Pacto se comprometen a respetar la libertad de los padres $y$, en su caso, de los tutores legales, de escoger para sus hijos o pupilos escuelas diferentes de las creadas por las autoridades públicas, siempre que aquellas satisfagan las normas minimas que el Estado prescriba o apruebe en materia de enseñanza, y hacer que sus hijos o pupilos reciban la educación religiosa o moral que esté de acuerdo con sus propias convicciones" (art. 13)

4. "Nada de lo dispuesto en este artículo se interpretará como una restricción de la libertad de los particulares y entidades para establecer y dirigir instituciones de enseñanza, a condición de que se respeten los principios enunciados en el párrafo y de que la educación en estas instituciones se ajuste a las normas minimas que prescriba el Estado" (art. 13).

\section{CONCLUSIONES}

1. LaNaciónperuanaexpresaensusconstituciones, particularmente las iniciales, un espíritu religioso consistente $\mathrm{y}$ fundamentalmente católico.

2. Las constituciones y leyes educativas se complementan, expresando con claridad la afirmación de la obligatoriedad de la enseñanza de la religión en toda la etapa escolar como forma de buscar la continuidad de ese legado en el tiempo.

3. La realidad educativa escolar en el Perú señala que los antiguos Planes y Programas, así como los modernos Currículos Escolares, han mostrado de manera consistente el cumplimiento de dicha exigencia constitucional y legal.

4. El sentido de que una educación integral era la unión de lo ético, cívico y religioso notorio en el primer siglo republicano, se ha ido debilitando en esos aspectos, aunque enriqueciendo en otros.

5. La aceptación de los derechos de otras confesiones a expresarse en lo escolar se ha ido reconociendo progresivamente, así como el respeto al derecho de conciencia de los padres de familia de no ser forzados en la formación religiosa de sus hijos.

\section{REFERENCIAS}

Basadre, J. (1822 a 1933). Historia de la República. 8 va edición. 16 volúmenes. Lima. Perú. Universidad Ricardo Palma.

Código de Derecho Canónico (1983). Pamplona. España. EUNSA.

Constituciones del Perú (1823, 1828, 1834, 1839, 1856, 1860, 1867, 1920, 1933, 1979, 1993). Lima. Perú

Constitución de la Monarquía Española (1812). Cádiz. España.

Leyes generales u orgánicas de Educación, o reglamentos de Instrucción del Perú: 1850, 1855, 1876, 1941, 1972, 1982, 2003. Lima. Perú.

Palomino, C. E. (2006). Politica Educativa escolar en el Perú. (2006). Lima. Perú.

Palomino, C. E. (2016). Revisando la Educación en el Perú del siglo XXI. 2016. Chiclayo. Perú.

Ugarte del Pino, V. (1970). Historia de las constituciones del Perú. Lima. Perú. 